\title{
Aneurisma subanular mitral: correção cirúrgica
}

\author{
Paulo M. PÊGO-FERNANDES*, Marisa AMATO*, Luíz Felipe P. MOREIRA*, Luís Alberto DALLAN*,
} Noedir A. G. STOLF*

\begin{abstract}
PÊGO-FERNANDES, P. M.; AMATO, M.; MOREIRA, L. F. P.; DALLAN, L. A.; STOLF, N. A. G. - Aneurisma subanular mitral: correção cirúrgica. Rev. Bras. Cir. Cardiovasc., 8(2): 163-166, 1993.

RESUMO: O aneurisma subanular mitral é doença pouco conhecida em nosso país. Foi descrita, inicialmente, e é vista com maior frequeência na população negra das regiões sul e oeste da África. Parece relacionar-se a uma fraqueza da parede ventricular na área de implantação da cúspide posterior da valva mitral, levando à insuficiência dessa valva. É relatado o caso de homem de 20 anos, branco, com queixa de dispnéia e taquicardia. Foi internado devido a evolução para classe funcional IV. Após compensação clínica, apresentava, ainda, sopro sistólico $(+++/++++)$ em área mitral. A radiografia de tórax mostrou aumento da área cardiaca $(+++/++++)$ às custas de átrio e ventrículo esquerdo. 0 ecocardiograma, além do aumento dessas câmaras, revelou dilatação aneurismática na regiāo posterior do ventriculo esquerdo, justa-valvar em relação à valva mitral, com sinais de incompetência severa, assim como a cineangiocardiografia. Submetido a cirurgia em 20/10/86, quando foi realizada ressecção do aneurisma, utilizando-se placas de pericárdio bovino para reconstrução. Foi feita, também, troca de valva mitral por válvula biológica. 0 paciente evoluiu bem, tendo recebido alta hospitalar no oitavo dia de pós-operatório. Atualmente, é assintomático, o ecocardiograma de controle revelou correção cirúrgica satisfatória.
\end{abstract}

DESCRITORES: aneurisma subanular mitral, cirurgia.

\section{INTRODUÇÃO}

O aneurisma subanular mitral é doença pouco conhecida em nosso país. Foi descrita, inicialmente, e é vista com maior freqüência na população negra das regiões sul e oeste da África ${ }^{1}$. Parece estar relacionada a uma fraqueza da parede ventricular na área de implantação do folheto posterior da valva mitral 1,6 .

O objetivo deste trabalho é o relato do caso de paciente jovem, branco, com aneurisma subanular mitral tratado cirurgicamente, com sucesso.

\section{RELATO DO CASO}

Paciente masculino, 20 anos, branco, foi hos- pitalizado em 1986 com queixa de dispnéia e taquicardia há um ano. A dispnéia era de caráter progressivo, sendo classificada como grau IV (NYHA), na internação.

Ao exame físico, o paciente apresentava-se em bom estado geral, afebril, hidratado, sem gânglios palpáveis, com pulso de 80 batimentos por minuto e pressão arterial de $130 \times 80 \mathrm{mmHg}$. O pulmão não apresentava anormalidades. Apresentava ictus pálpavel e desviado para linha axilar anterior; à ausculta, a primeira bulha hipofonética com sopro sistólico $(+++/++++)$, em área mitral, se irradiava para todo o precórdio. O abdome apresentava-se com ruídos hidroaéreos normais, fígado percutível não palpável e baço não percutível nem palpável.

Aos raios $\mathrm{X}$ havia aumento da área cardíaca

Trabalho realizado no Instituto do Coração do Hospital das Clínicas da Faculdade de Medicina da Universidade de São Paulo. São Paulo, SP, Brasil. Recebido para publicaçăo em 20 de junho, 1993.

- Do Instituto do Coração do Hospital das Clínicas da Faculdade de Medicina da Universidade de Săo Paulo.

Endereço para separatas: Paulo Pêgo-Fernandes. Hospital Jaraguá. Rua Juriti, 73. 04520-000 São Paulo, SP. 
PÊGO-FERNANDES, P. M.; AMATO, M.; MOREIRA, L. F.P.; DALLAN, L. A.; STOLF, N. A. G. - Aneurisma subanular mitral: correçăo cirúrgica. Rev. Bras. Cir. Cardiovasc., 8(2): 163-166, 1993.

$(+++/++++)$, às custas do átrio e ventrículo esquerdo. Apresentava deformidade de silhueta cardíaca. O eletrocardiograma mostrava ritmo sinusal, sobrecargas atrial e ventricular esquerda. 0 ecocardiograma revelou dilatação moderada de átrio esquerdo e acentuada de ventrículo esquerdo. Evidenciou, também, dilatação aneurismática na região posterior do ventrículo esquerdo, justa-valvar em relação à valva mitral, com sinais de incompetência significativa (Figura 1).

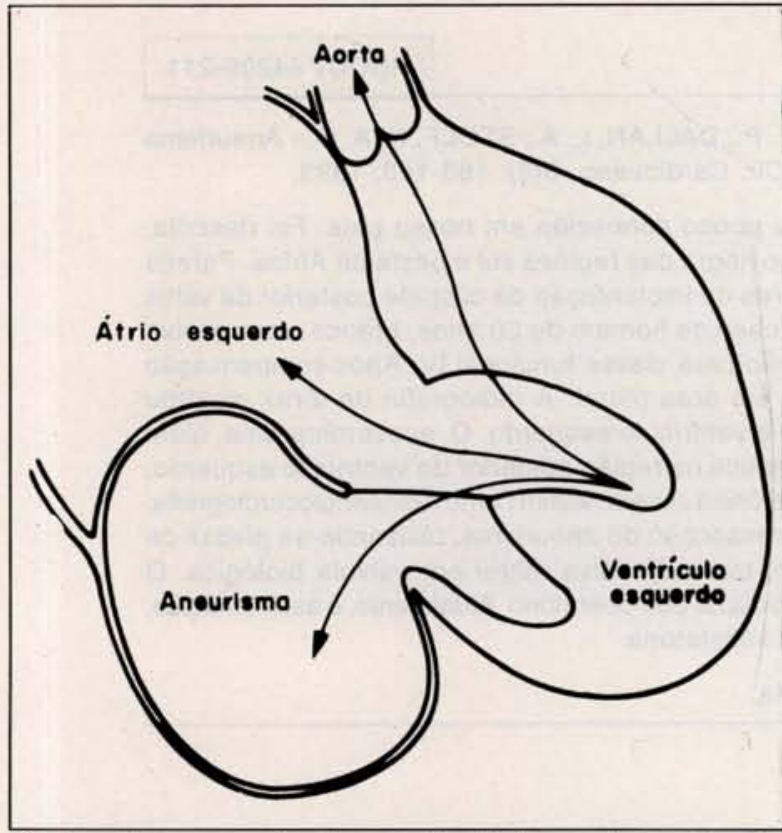

Fig. 1. Esquema mostrando o grande colo do aneurisma subanula mitral, $e$ a regurgitação mitral.

o cateterismo evidenciou um divertículo cardíaco com insuficiência mitral grave; à manometria apresentou elevação da pressão diastólica final de ventrículo esquerdo, elevação da pressão capilar pulmonar e hipertensão moderada em câmaras direitas.

Com diagnóstico de aneurisma subanular mitral com insuficiência mitral severa, foi indicada cirurgia. No dia 20/10/86 foi realizada ressecção do aneurisma, utilizando-se placas de pericárdio bovino para reconstrução (Figuras 2 e 3). Foi feita, também, troca de valva mitral para válvula biológica, devido a deformação e insuficiência.

O paciente evoluiu bem, recebendo alta hospitalar no $8^{\circ}$ dia de pós-operatório. Atualmente, encontra-se assintomático, no $6^{\circ}$ ano de pós-operatório, revelando correção cirúrgica satisfatória.

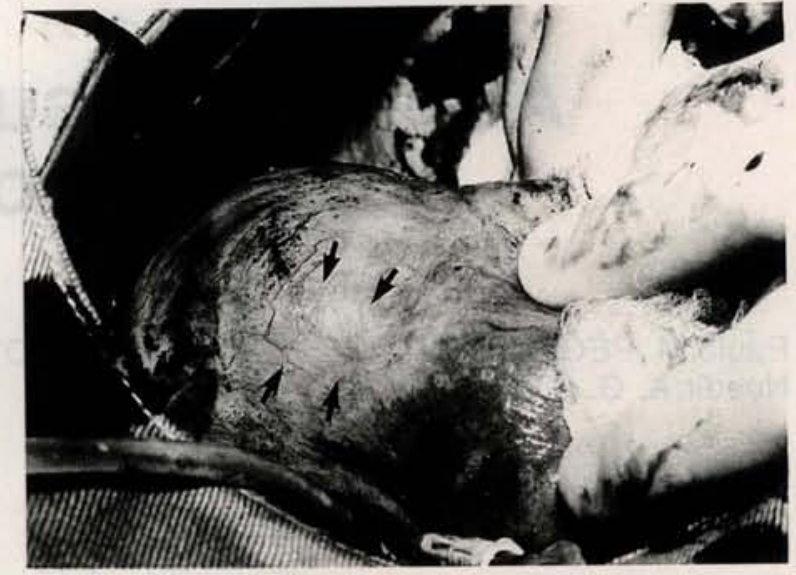

Fig. 2 - Foto cirúrgica mostrando a vista externa do aneurisma subanular mitral (setas)

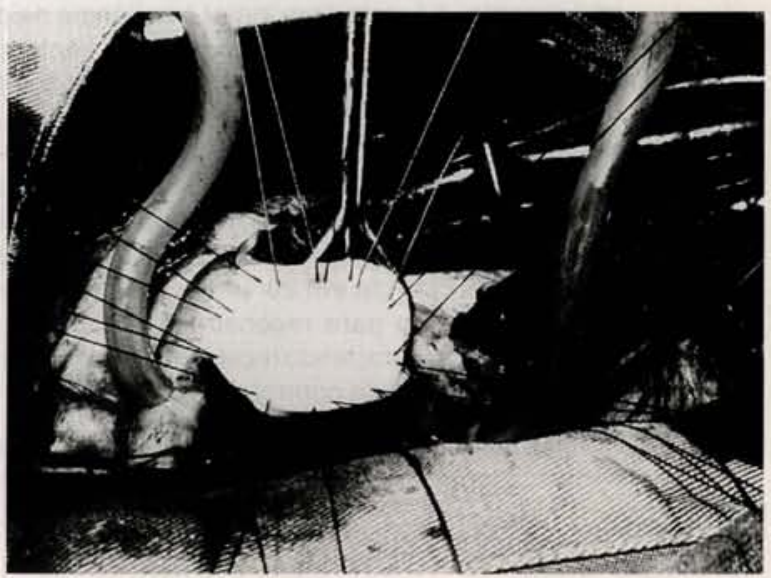

Fig. 3 - Foto cirúrgica mostrando a placa de pericárdio bovino com pontos passados, para o fechamento do colo do aneurisma.

\section{COMENTÁRIOS}

A maioria dos aneurismas de ventrículo esquerdo encontrados na população adulta é secundária a cardiopatia isquêmica. Outras causas raras incluem sífilis, tuberculose, endocardite, sarcoidose e trauma.

Em nosso meio, a doença de Chagas contribuiu com maior porcentual 3 .

O aneurisma subanular mitral deve ser diferenciado das outras formas de aneurisma do ventrículo esquerdo, especialmente daqueles de origem isquêmica. Para essa diferenciação, história, sorologia, antecedentes, alterações eletrocardiográficas e grupo étnico devem ser levados em consideração. O nosso paciente apresentava sorologias negativas, jovem, sem antecedentes clinicos ou sinais eletrocardiográficos de isquemia miocárdica prévia. 
PÊGO-FERNANDES, P. M.; AMATO, M.; MOREIRA, L. F.P.; DALLAN, L. A.; STOLF, N. A. G. - Aneurisma subanular mitral: correção cirúrgica. Rev. Bras. Cir. Cardiovasc., 8(2): 163-166, 1993.

A localização do aneurisma justa-aparelho valvar mitral freqüentemente resulta em regurgitação valvar causada por perda de suporte, distorção e prolapso de folheto posterior. Em nosso paciente, os sinais clínicos eram típicos de insuficiência mitral severa. Outra característica encontrada em nosso caso é a presença de multiloculações na cavidade do aneurisma.

Outro ponto que merece comentários é a nomenclatura. Atualmente, o termo " aneurisma subanular mitral" tem tido maior aceitação. Porém, há autores que classificam essa afecção junto aos divertículos cardíacos. Por definição, divertículo é de origem congênita, tem o colo estreitado e seuas paredes são constituídas por músculo ou fibrose ${ }^{4}$. Divide-se em três tipos: um deles, localiza-se na base, possui paredes fibrosadas e associa-se com insuficiência mitral ou aórtica (dependendo da sua localização), ocorrendo mais em negros africanos ${ }^{4}$. Preferimos, neste trabalho, o uso da denominação "aneurisma subanular mitral", assim como outros autores $1-3,5,6$, por considerarmos que se trate de afecção específica.

Uma complicação descrita no tratamento cirúrgico é o infarto agudo do miocárdio. Esse fato se deve à proximidade da artéria circunflexa da borda do aneurisma. Com a sutura do colo pode-se, inadvertidamente, ligar a coronária, se não se tiver isso em mente ${ }^{2}$.
O tratamento cirúrgico é o único método de tratamento desta condição, mas levava a mortalidade significativa, nas experiências iniciais ${ }^{2}$.

ANTUNES ${ }^{2}$, que tem grande experiência cirúrgica com a afeç̧ão, relatou, em 1987, a correção através do átrio, ao invés da abordagem através do aneurisma. Nessa ocasião, relatou 9 pacientes operados por essa abordagem, em que o colo do aneurisma foi fechado diretamente. Nessa casuística, em 4 doentes fez valvuloplastia com anel de Carpentier. Não foi necessária a troca valvar nos 9 doentes, e o autor creditou esse fato ao acesso transatrial. No nosso caso, abordamos o aneurisma através do átrio, de modo semelhante ao descrito por Dr. Antunes, porém, devido ao grande colo do aneurisma, preferimos o fechamento com placa de pericárdio bovino. Como a valva estava muito deformada, optamos pela substituição por válvula de pericárdio bovino.

Acreditamos que o diagnóstico preciso da afecção, através da história clínica, do ecocardiograma e/ou da cineangiocardiografia, propicia o planejamento cirúrgico adequado. Com esse planejamento, o risco cirúrgico é baixo e plenamente aceitável, justificando a intervenção em pacientes sintomáticos.

RBCCV 44205-211

PÊGO-FERNANDES, P. M.; AMATO, M.; MOREIRA, L. F. P.; DALLAN, L. A.; STOLF, N. A. G. - Submitral left ventricular aneurysm: surgical correction. Rev. Bras. Cir. Cardiovasc., 8(2):162-165, 1993.

ABSTRACT: Submitral left ventricular aneurysm is a poorly known entity in our country; it has been described mainly in the southern and western African black populations. It appears to be related to a ventricular wall weakness in the area of implantation of the posterior mitral valve leaflet. A 20-year-old white man was admitted with complaints of dyspnea and palpitation. On admission he was in NYHA class IV. After medical treatment the cardiac area was very enlarged and there was a systolic murmur +++/++++. The twodimensional echocardiography as well as the angiocardiography revealed annular submitral left ventricular aneurysm associated with severe mitral regurgitation. He was operated upon on October 20, 1986. We closed the neck of the aneurysm with a pericardial patch. The mitral valve was replaced due to a severe distortion. The patient is well, six years after the surgery.

DESCRIPTORS: aneurysms, left ventricle, submitral, surgery. 
PÊGO-FERNANDES, P. M.; AMATO, M.; MOREIRA, L. F.P.; DALLAN, L. A.; STOLF, N. A. G. - Aneurisma subanular mitral: correçåo cirúrgica. Rev. Bras. Cir. Cardiovasc., 8(2): 163-166, 1993.

\section{REFERÊNCIAS BIBLIOGRÁFICAS}

1 ABRAHANS, G.; BARTON, C. J.; COCKSHOTT, W. P.; EDINGTON, G. M.; WEAVER, E. J. M.- Annular subvalvular left ventricular aneurysms. Q. J. Med., 31: 345-360, 1962.

2 ANTUNES, M. J. - Submitral left ventricular aneurysms. J. Thorac. Cardiovasc. Surg., 94: 241-245, 1987.

BRITO, J. C.; CARVALHO, H. G.; FEITOSA, G.; RIBEIRO, A. C.; NERY, A.; TADEU, E.; TORREÃO, J.; FIGUEIREDO, G.; FERREIRA Jr., A.; ELOY, R.; RIBEIRO, N. A. M. - Aneurisma anular subvalvar mitral do ventrículo esquerdo: apresentação de quatro casos. Arq. Bras. Cardiol., 45: 257-262, 1985.
4 GUERON, M.; HIRCH, M.; OPSCHITZER, I.; MOGEL, $P$. - Left ventricular diverticulum and mitral incompetence in asymptomatic children. Circulation, 53: 181-186, 1976.

5 GUIMARÃES, A. C.; SANTOS F. A.; ESTEVES, J. P.; ABREU, W. N.; VINHAES, L. A.; SOUZA, J. A.; MACHADO, A. - Annular subvalvular left ventricular aneurysms in Bahia, Brazil. Br. Heart J., 38: 1080$1085,1976$.

6 WOLPOWITZ, A.; ARMAN, B.; BARNARD, M. S.; BARNARD, C. N. - Annular subvalvular idiopatic left ventricular aneurysms in the black African. Ann. Thorac. Surg., 27: 350-355, 1978. 